\title{
New horizons in hypoxia signaling pathways in health and disease.
}

Peter J. Ratcliffe

University of Oxford and The Francis Crick Institute, London

Animal cells deploy a set of 2-oxoglutarate dependent dioxygenases to signal oxygen levels in cells by modulating the catalytic rate of post-translational hydroxylation of specific amino acids in hypoxia inducible factors (HIFs). HIFs transduce these signals by activation of extensive cellular and systemic responses when oxygen levels are low. Though this integrated pathway appears to be specific to animal systems, many components are represented more widely in life. It is now clear that all four eukaryotic kingdoms use catalytic protein oxidation and degradation to regulate gene expression in accordance with oxygen availability. These findings have opened new fields of biochemical, evolutionary, physiological research into hypoxia. The lecture will review recent advances in these areas. It will also review the opportunities and challenges for therapeutic modulation of the human oxygen sensing system in hypoxic diseases. 